\title{
Lo que decimos sobre la escritura: Caracterización de los recursos educativos digitales compartidos por centros y programas de escritura de Latinoamérical
}

Karen Shirley López Gil2

Pontificia Universidad Javeriana - Cali

Artículo de Investigación

Recibido: Marzo 30 de 2016 Aprobado: Mayo II de 2016

\section{Resumen}

Este artículo busca caracterizar los recursos educativos digitales compartidos por las páginas web de los centros y programas de escritura de Latinoamérica. Se realizó un estudio descriptivo transeccional con una muestra representativa de 3/4 recursos disponibles en la red. A través de una rejilla, se identificaron los propósitos de los recursos, sus temas, formatos, modalidades de representación de la información, público al que se dirigen, estrategias discursivas usadas en las explicaciones, enlaces

I Este artículo presenta resultados parciales del proyecto de investigación Análisis de los abordajes conceptuales, las metodologías de enseñanza-aprendizaje y las dinámicas de gestión académica de programas y centros de escritura de Latinoamérica, aprobado en la convocatoria Capital Semilla (20I5) de la Pontificia Universidad Javeriana Cali (Colombia), código 00004081. Este proyecto fue liderado por Violeta Molina Natera y Karen Shirley López Gil. Inició en enero de 2015 y finalizó en enero de 2016.

2 Directora del Centro de Escritura Javeriano. Docente del Departamento de Comunicación y Lenguaje de la Pontificia Universidad Javeriana Cali. Miembro del grupo de investigación Procesos y Medios de Comunicación. Psicóloga, fonoaudióloga, magíster en lingüística y español (Universidad del Valle, Colombia), máster en innovación e investigación educativa (UNED, España). Estudios de Doctorado en Educación (UNED, España). Contacto: Pontificia Universidad Javeriana Cali. Edificio El Samán, oficina 3.33. Correo electrónico: karens@javerianacali.edu.co 
a otros centros y programas, referencias bibliográficas, entre otras características. La identificación de estos aspectos anima a una reflexión sobre las representaciones y prácticas de escritura académica que se promueven desde estas publicaciones y las posibilidades de trabajar como red en la consolidación de recursos educativos digitales abiertos que fortalezcan la oferta formativa en línea sobre escritura.

Palabras clave: centros y programas de escritura, escritura académica, recursos educativos digitales, Internet, universidad, formación.

\title{
What we say about writing: characterization of digital educational resources shared by writing centers and programs in Latin America
}

\begin{abstract}
This article aims at characterizing the digital educational resources shared by the web pages of Latin American writing centers and programs. A cross-sectional study with a representative sample of 314 resources available on the net was carried out. By means of the use of a chart, the purposes of such resources were identified as well as their topics, formats, modalities for the representation of information, target public, discourse strategies used in explanations, links to other centers and programs, and bibliographic references, among other characteristics. The identification of such aspects drives to a reflection about the academic writing representations and practices that are promoted by these sites and about the possibility of working as a network in the strengthening of open digital educational resources that enhance the supply of online writing educational programs.
\end{abstract}

Key words: writing centers and programs, academic writing, digital educational resources, the Internet, university, education.

\section{O que dissemos sobre a escrita: caracterização dos recursos digitais compartilhados por centros e programas de escrita em América Latina}

\section{Resumo}

Este artigo busca caracterizar os recursos educativos digitais compartilhados pelas páginas web dos centros urbanos e programas de escrita de América Latina. Foi realizado um estudo descritivo transseccional com uma amostra representativa de 314 recursos disponíveis na rede. A traves de uma grade foram identificados os fins dos recursos, seus temas, formatos, modalidades de representação da informação, público ao qual estão dirigidos, estratégias discursivas usadas nas explicações, enlaces a outros centros e programas, referências bibliográficas, entre outras características. A identificação de estes aspectos estimula uma reflexão sobre as representações e práticas de escrita acadêmica que são promovidas por estas publicações e as possibilidades de trabalhar com rede na consolidação de recursos educativos digitais abertos que fortaleçam a oferta formativa em linha sobre a escrita.

Palavras chave: centros e programas de escrita, escrita acadêmica, recursos educativos digitais, Internet, universidade, formação. 


\section{Introducción}

En los últimos 10 años se ha evidenciado un crecimiento de estrategias institucionales para apoyar la escritura en las universidades de Latinoamérica, lo que ha fomentado la creación de centros y programas de escritura ${ }^{3}$. Estos espacios institucionales suelen usar distintas estrategias para acompañar a los estudiantes en el desarrollo de sus competencias escriturales, entre ellas se encuentran las tutorías presenciales y virtuales, el trabajo colaborativo con docentes de otras disciplinas, el diseño y coordinación de cursos y la producción de recursos sobre escritura ${ }^{4}$. La producción de recursos educativos parece ser una tendencia de los centros y programas de América Latina ${ }^{5}$, como también lo ha sido en Estados Unidos, en donde muchos centros disponen de páginas web con materiales que los usuarios pueden completar sin la mediación de un tutor ${ }^{6}$.

Aunque los recursos no suelen ser el principal servicio de los centros y programas, - con algunas excepciones como el Centro de Recursos para la Escritura Académica (CREA) del Tecnológico de Monterrey-, su disponibilidad en la red implica que pueden ser consultados por un público amplio y, por consiguiente, pueden tener una incidencia en las representaciones y prácticas de escritura académica de los usuarios. De igual forma, estamos en un momento histórico en el que se apuesta por el aprendizaje autodirigido y en el que los usuarios tienen altas expectativas respecto a la calidad de los materiales que encuentran en línea ${ }^{7}$, pues Internet es concebida como una gran biblioteca abierta que contiene toda la información que se necesita para responder a las demandas en el contexto educativo.

Por estas razones, es fundamental identificar cuáles son las características de los recursos digitales que están compartiendo públicamente los centros y programas de escritura en Latinoamérica y reflexionar sobre su pertinencia. Esta identificación se puede constituir en una oportunidad para que los centros y programas de la región trabajen en red y consoliden una oferta de recursos educativos abiertos que potencien sus posibilidades formativas.

3 Molina, Violeta. Panorama de los centros y programas de escritura en Latinoamérica. Cali, Colombia: Sello Editorial Javeriano, 2015.

4 Núñez, Juan. "Una aproximación a los centros de escritura en Iberoamérica”. Revista Legenda, vol. I7, n 7 (20 I 3), págs.62- 102.

5 Narváez, Elizabeth. Iniciativas de enseñanza de la escritura en países latinoamericanos: Tendencias emergentes de las páginas web de centros de escritura y programas de escritura, 2013. Memorias del I Congreso Latinoamericano de Centros de Escritura. Recuperado de: https://sites.google.com/site/congresocentrosdeescritura/file-cabinet

6 Martínez, Diane \& Olsen, Leslie. "Online Writing Labs" (p. I83-210). In Hewett, Beth and DePew, Kevin (Eds.) Foundational Practices of Online Writing Instruction. Perspectives on Writing. Fort Collins, Colorado: The WAC Clearinghouse and Parlor Press, 2015.

7 Blair, Kristine. "Teaching Multimodal Assignments in OWI Contexts" (p. 47I-49I). In Hewett, Beth and DePew, Kevin (Eds.) Foundational Practices of Online Writing Instruction. Perspectives on Writing. Fort Collins, Colorado: The WAC Clearinghouse and Parlor Press, 2015. 


\section{Recursos educativos digitales: características y tipología}

Los recursos educativos digitales pueden entenderse como cualquier material que ha sido creado con un propósito formativo y que se encuentra disponible en medios electrónicos ${ }^{8}$. Estos materiales atienden a distintos temas, formatos y condiciones de uso, y pueden ser utilizados por docentes y estudiantes de cualquier nivel educativo e, incluso, por usuarios que no se encuentran en sistemas educativos formales.

Los recursos educativos se caracterizan por organizar los contenidos de manera que el usuario puede apropiarse de ellos de forma autónoma, sin necesidad de contar con la mediación de un tutor. Aunque un recurso puede pertenecer a un conjunto más amplio, debe tener características de completitud, unidad y suficiencia, pues su disponibilidad en la web abierta puede llevar a que cualquier internauta use ese material para el aprendizaje autogestionado.

A continuación, se presenta una breve clasificación de los recursos educativos digitales:

\begin{tabular}{|c|c|}
\hline Por permisos de distribución y uso: & Por formato de información digital: \\
\hline Privado & Textual \\
Público & Sonoro \\
Abierto & Visual \\
\hline Por tipo de interacción con el usuario: & Audiovisual \\
\hline Activo & Multimedial \\
\hline Expositivo & \\
\hline Combinado & \\
\hline
\end{tabular}

Tabla I. Clasificación de los recursos educativos digitales. Elaboración a partir de la propuesta del Ministerio de Educación Nacional de Colombia, 2012.

De acuerdo con las condiciones de distribución y uso, los recursos educativos digitales pueden clasificarse en privados, públicos y abiertos?. Los recursos de acceso privado son aquellos que están disponibles en la red, pero su uso está restringido a ciertos grupos; están protegidos por Copyright y no pueden ser modificados ni reutilizados sin permisos. Generalmente, su utilización requiere de algún pago. Los recursos de acceso público son aquellos a los que se puede acceder de forma gratuita, pero tienen algunas restricciones en los permisos de modificación o adaptación; suelen tener permisos Copyleft ${ }^{10}$

8 UNESCO. Declaración de París de 2012 sobre los REA. 2012. Recuperado de: http://www.unesco.org/new/fileadmin/MULTIMEDIA/ $\mathrm{HQ} / \mathrm{Cl} / \mathrm{Cl} / \mathrm{pdf} /$ Events/Spanish_Paris_OER_Declaration.pdf

9 Ministerio de Educación Nacional de Colombia. Recursos educativos digitales abiertos. Bogotá: Colección Sistema Nacional de Innovación Educativa con uso de TIC, 2012.

I0 Copyleft se refiere a las prácticas que permiten la distribución de copias, modificaciones y adaptaciones de las obras, con menos restricciones que Copyright. 
a través de licencias como Creative Commons. Por su parte, los recursos educativos abiertos, además de ser públicos y de acceso libre, otorgan a los usuarios permisos para derivar, modificar o adaptar los contenidos sin que esto implique ningún costo o devenga en problemas de uso legal de la información.

Los recursos educativos digitales también pueden clasificarse por el formato que privilegian en la comunicación y organización de la información'". De esta manera, encontramos recursos textuales, en los que predomina el sistema de representación escrito, aunque pueden complementarse con algunos elementos gráficos. Los recursos sonoros presentan información predominantemente en un canal acústico. Los recursos visuales privilegian la representación gráfica de la información. Los recursos audiovisuales integran información en las modalidades textuales, sonoras y visuales. Por su parte, los recursos multimediales integran múltiples formatos: textuales, sonoros, visuales y audiovisuales, y permiten una mayor interacción con el usuario.

De acuerdo con el tipo de interacción que se promueve, los recursos se pueden clasificar como activos, cuando favorecen el aprendizaje del usuario mediante la manipulación del material, la toma de decisiones y la inclusión de respuestas; expositivos, cuando permiten que el usuario navegue, visualice y lea la información; o combinados, cuando mezclan los otros dos tipos de interacción ${ }^{12}$. Barbera y Rochera ${ }^{13}$ plantean una clasificación similar, en la que cruzan las variables de producción/reproducción de información con los propósitos de los recursos: informativos/participativos.

Las autoras definen los materiales reproductivos-informativos como aquellos en los que se presentan contenidos para que el usuario los consulte, desde una visión pasiva de "recepción de información". Los materiales reproductivos-participativos permiten a los aprendices interactuar con la información a través de ejercicios puntuales con realimentaciones cerradas, entre estos son comunes los ejercicios de ortografía y gramática. Los materiales productivo-informativos son aquellos en los que el usuario accede a una información y genera una respuesta abierta como un escrito o una grabación de voz. Los materiales productivo-participativos, por su parte, ofrecen contenidos flexibles que se van ajustando a las respuestas y necesidades de los usuarios.

Como puede evidenciarse, los recursos educativos digitales son un conjunto muy amplio de materiales, con características y propósitos muy diversos. Pueden clasificarse también por sus intencionalidades, por el tipo de aprendizaje que promueven, por el público al que se dirigen, por niveles educativos, temas, etc.

I Ministerio de Educación Nacional de Colombia. Recursos educativos digitales abiertos. Bogotá: Colección Sistema Nacional de Innovación Educativa con uso de TIC, 2012.

I2 Ministerio de Educación Nacional de Colombia. Recursos educativos digitales abiertos. Bogotá: Colección Sistema Nacional de Innovación Educativa con uso de TIC, 2012.

I3 Barbera, Elena y Rochera, María José. "Los entornos virtuales de aprendizaje basados en el diseño de materiales autosuficientes y el aprendizaje autodirigido" (pág. 173-193). En Coll, César y Monereo, Carles (Eds.) Psicología de la educación virtual. Madrid: Ediciones Morata, 2008. 


\section{Metodología}

Se realizó un estudio descriptivo de corte transeccional que buscaba caracterizar los recursos digitales publicados por las páginas web de los centros y programas de escritura de Latinoamérica. La identificación de los recursos se llevó a cabo en el periodo junio-octubre de 2015.

Inicialmente, se identificaron las páginas web a través de la Red Latinoamericana de Centros y Programas de Escritura ${ }^{14}$. Posteriormente, se hicieron otras búsquedas en Internet y se identificaron un total 4 I centros y programas en la región. De los 4 I identificados, 34 contaban con página web y 17 de esas páginas web compartían recursos educativos sobre escritura.

Se identificó un universo de 585 recursos digitales, atendiendo a criterios de completitud (abordaje de una temática específica e identificación con un título) y de accesibilidad (acceso público o abierto). Se seleccionó una muestra representativa de 314 recursos, con nivel de confianza de 99\% e intervalo de confianza de $5^{15}$. Se realizó un muestreo aleatorio estratificado, es decir, se seleccionaron al azar los recursos en cada página, guardando una proporción entre la cantidad de recursos publicados y la cantidad de recursos tomados para la muestra representativa. La cantidad de recursos caracterizados por página se especifican en la siguiente tabla:

\begin{tabular}{|c|c|c|}
\hline Páginas web & $\mathrm{F}$ & $\%$ \\
\hline Blog de redacción del Programa de Comunicación Académica de la Pontificia Universidad Católica del Perú & 72 & 22,9 \\
\hline Centro de Escritura Javeriano de la Pontificia Universidad Javeriana Cali & 38 & 12,1 \\
\hline Centro de Lectura y Redacción de la Universidad del Turabo & 27 & 8,6 \\
\hline Centro de Escritura Académica y Pensamiento Crítico de la Universidad de las Américas de Puebla & 23 & 7,3 \\
\hline Centro de Redacción Multidisciplinario de la Universidad Interamericana de Puerto Rico & 23 & 7,3 \\
\hline Centro de Escritura Universitaria de la Universidad de San Andrés & 22 & 7 \\
\hline Centro de Recursos para la Escritura Académica (CREA) del Tecnológico de Monterrey & 21 & 6,7 \\
\hline Programa de Escritura Centro de Español de la Universidad de los Andes & 20 & 6,4 \\
\hline Centro de Aprendizaje, Redacción y Lenguas del Instituto Tecnológico Autónomo de México & 15 & 4,8 \\
\hline Centro de Escritura de la Universidad del Cauca & 11 & 3,5 \\
\hline Centro Virtual de Escritura de la Universidad de Buenos Aires & 9 & 2,9 \\
\hline
\end{tabular}

14 Red Latinoamericana de Centros y Programas de Escritura. Integrantes. 2015. Consultado 25 de mayo de 2015 Recuperado de https://sites.google.com/site/redlacpe/integrantes

I5 Nivel de confianza: probabilidad de que el parámetro de población se encuentre dentro del intervalo de confianza; en este caso, esta probabilidad es de 99\%. Intervalo de confianza: rango que muestra los límites de confianza de una proporción, dado por un recorrido o desviación, en este estudio \pm 5 . 


\begin{tabular}{|c|c|c|}
\hline Páginas web & F & $\%$ \\
\hline DIGA-Centro de apoyo para la lectura, la oralidad y la escritura del Centro de Estudios Superiores de Administración & 9 & 2,9 \\
\hline Centro de Recursos para el Éxito Estudiantil - CREE y programa Eficacia Comunicativa de la Universidad del Norte & 9 & 2,9 \\
\hline Centro de Escritura de la Universidad Popular Autónoma del Estado de Puebla & 8 & 2,5 \\
\hline Programa de Lectura y Escritura Académicas de la Pontificia Universidad Católica de Chile & 5 & 1,6 \\
\hline Centro de Escritura CEDES de la Universidad Católica del Uruguay & I & 0,3 \\
\hline $\begin{array}{l}\text { Programa de desarrollo de habilidades de lectura y escritura académica a lo largo de la carrera (PRODEAC) de la } \\
\text { Universidad Nacional de General Sarmiento }\end{array}$ & I & 0,3 \\
\hline Total & 314 & 100,0 \\
\hline
\end{tabular}

Tabla 2. Cantidad de recursos analizados en cada página web. Fuente propia.

Para caracterizar los recursos educativos, se construyó una rejilla de análisis, apoyada en instrumentos usados en otras investigaciones (Pinto, 201016; Pinto y Gómez-Camarero, 201 177; Colectivo Educación Infantil y TIC, 201418; Lamoroux, 201419 ), y se realizó un pilotaje en el que se revisaron 17 recursos digitales públicos, uno por cada página web. Se hicieron modificaciones que permitieran que el instrumento se ajustara a la diversidad de características de los recursos estudiados (ver anexo I). Los resultados fueron procesados con apoyo del SPSS v.22.

\section{Resultados}

Los 314 recursos analizados en la muestra son de acceso gratuito y se encuentran disponibles en la red. $98 \%$ están escritos en español y $2 \%$ en inglés. Los recursos que están en inglés pertenecen a centros o programas de universidades de Puerto Rico y México, en las que suele haber comunicación en ambas lenguas. Los 17 centros y programas que comparten recursos a través de sus páginas web están ubicados en Colombia (5), México (4), Argentina (3), Puerto Rico (2), Chile (I), Uruguay (I) y Perú ( I).

A continuación, se analizan los datos recogidos a través de la rejilla, a la luz del marco teórico y de los antecedentes investigativos sobre recursos educativos digitales y sobre escritura académica.

I6 Pinto, María. "Evaluación y mejora de la calidad de los recursos educativos electrónicos en el ámbito universitario español desde un enfoque documental". Ibersid, vol. 4 (20 I0), págs. I05- I 16.

17 Pinto, María y Gómez-Camarero, Carmen. "Propuesta de criterios e indicadores internacionales para la evaluación de los recursos educativos electrónicos". Ibersid, vol. 5 (201 I), págs. 81-87.

I8 Colectivo Educación Infantil y TIC. Recursos educativos digitales para la educación infantil (REDEI). Revista Zona Próxima, $n^{\circ} 20$, enero-junio (20|4), págs. I-2I.

19 Lamorux, Tomas. Recursos audiovisuales en Internet diseñados para el aprendizaje del francés como lengua extranjera (FLE) dirigido a un público adulto. Tesis doctoral. España: Universidad Nacional de Educación a Distancia, 2014. 


\section{Tipos de recursos de acuerdo con los propósitos formativos}

Esta categoría hace referencia a las intencionalidades formativas de los recursos.

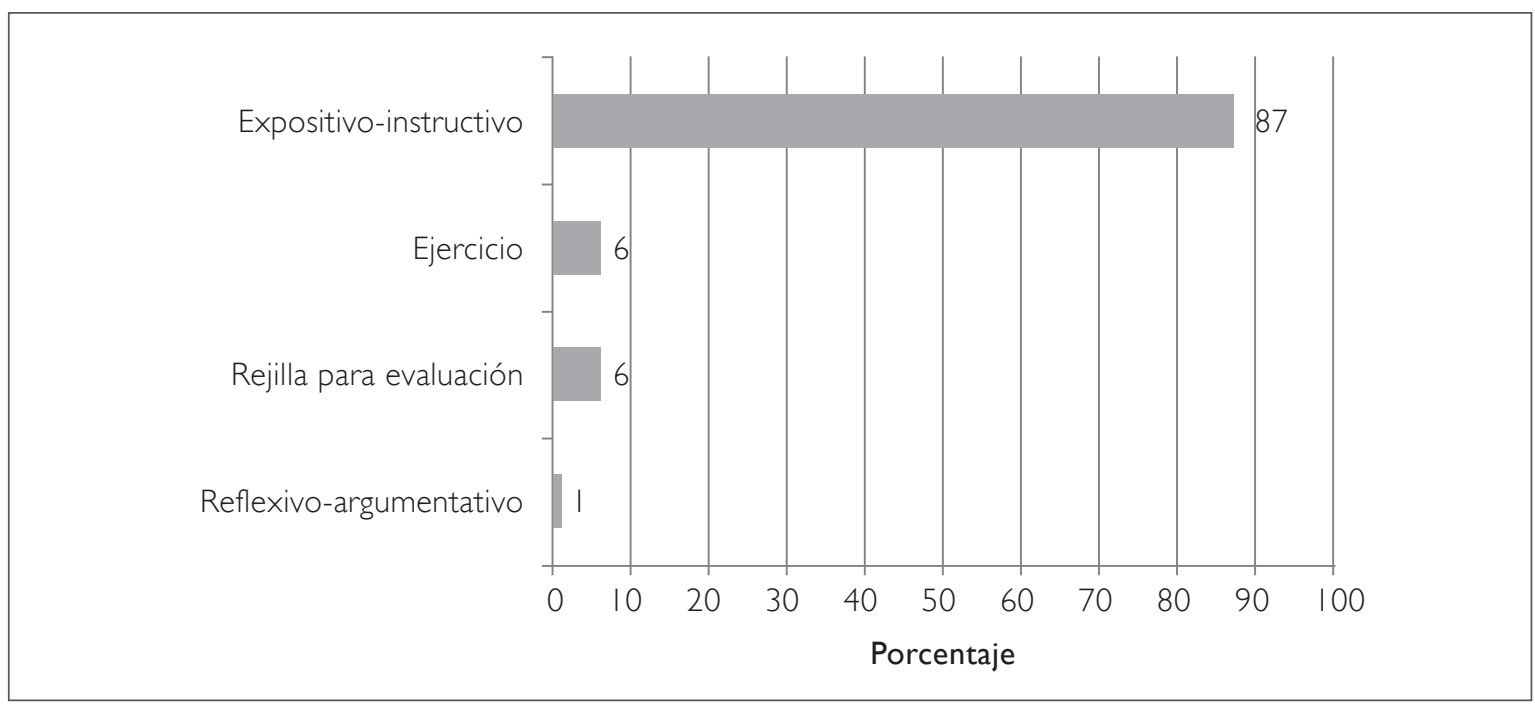

Gráfico I. Tipos de recursos de acuerdo con el propósito formativo. Fuente propia.

Como se muestra en el gráfico I, el $87 \%$ de los recursos (273) corresponde a propósitos explicativos-instructivos. Estos materiales se caracterizan por orientar a los usuarios sobre cómo desarrollar un procedimiento, por ejemplo, cómo planear un texto, cómo citar, cómo escribir un texto argumentativo, cómo escribir un resumen, etc. Dentro de este grupo de recursos, se identificaron como estrategias discursivas para explicar los contenidos ${ }^{20}$ : definición/descripción más ejemplo (40\%), definición/descripción (29\%), comparación/contraste (8\%), fases/procesos (6\%), ilustración (6\%), clasificación (6\%), ejemplo y ejercicio (6\%).

Se evidencian algunos recursos que plantean ejercicios prácticos (6\%), en su mayoría, sobre citación, ortografía y puntuación. $2 \%$ de estos ejercicios son interactivos, es decir, permiten que el usuario proporcione una respuesta (cerrada) y ofrecen realimentación. En esta categoría se destacan los ejercicios interactivos sobre aspectos formales del blog de redacción del Programa de Comunicación Académica de la Pontificia Universidad Católica del Perú y la página del Centro de Aprendizaje, Redacción y Lenguas del ITAM.

20 Clasificación apoyada en la propuesta de Álvarez, Teodoro y Ramírez, Roberto. "El texto expositivo y su escritura". Revista Folios, julio-diciembre (2010), págs. 73-88 
Las rejillas (6\%), que aparecen también con los nombres de rúbricas o matrices, proponen criterios y niveles de desempeño para evaluar las producciones escritas. Están dirigidas principalmente a docentes, pero también pueden ser usadas por los estudiantes en procesos de autoevaluación de los textos. Algunos ejemplos de rejillas se encuentran en las páginas del Centro de Español de la Universidad de los Andes, el Centro de Lectura y Redacción de la Universidad del Turabo y el Centro de Redacción Multidisciplinario de la Universidad Interamericana de Puerto Rico.

En la muestra, aparecen apenas 3 recursos reflexivo-argumentativos (1\%), en los que se busca sustentar la importancia de la lectura y la escritura y se plantean algunas reflexiones sobre las implicaciones que tienen estos procesos en la universidad. Estos recursos se dirigen a un público docente.

\section{Contenidos de los recursos}

Esta categoría hace referencia a los temas que se abordan en los recursos. Para su análisis, se tuvo en cuenta el título asignado al material, así como los contenidos y la estructura interna, ya que se encontraron importantes variaciones en los nombres de los recursos, aun cuando sus contenidos y referencias bibliográficas eran equivalentes.

Los temas de los 314 recursos fueron agrupados en 12 categorías, como se muestra en el gráfico 2:

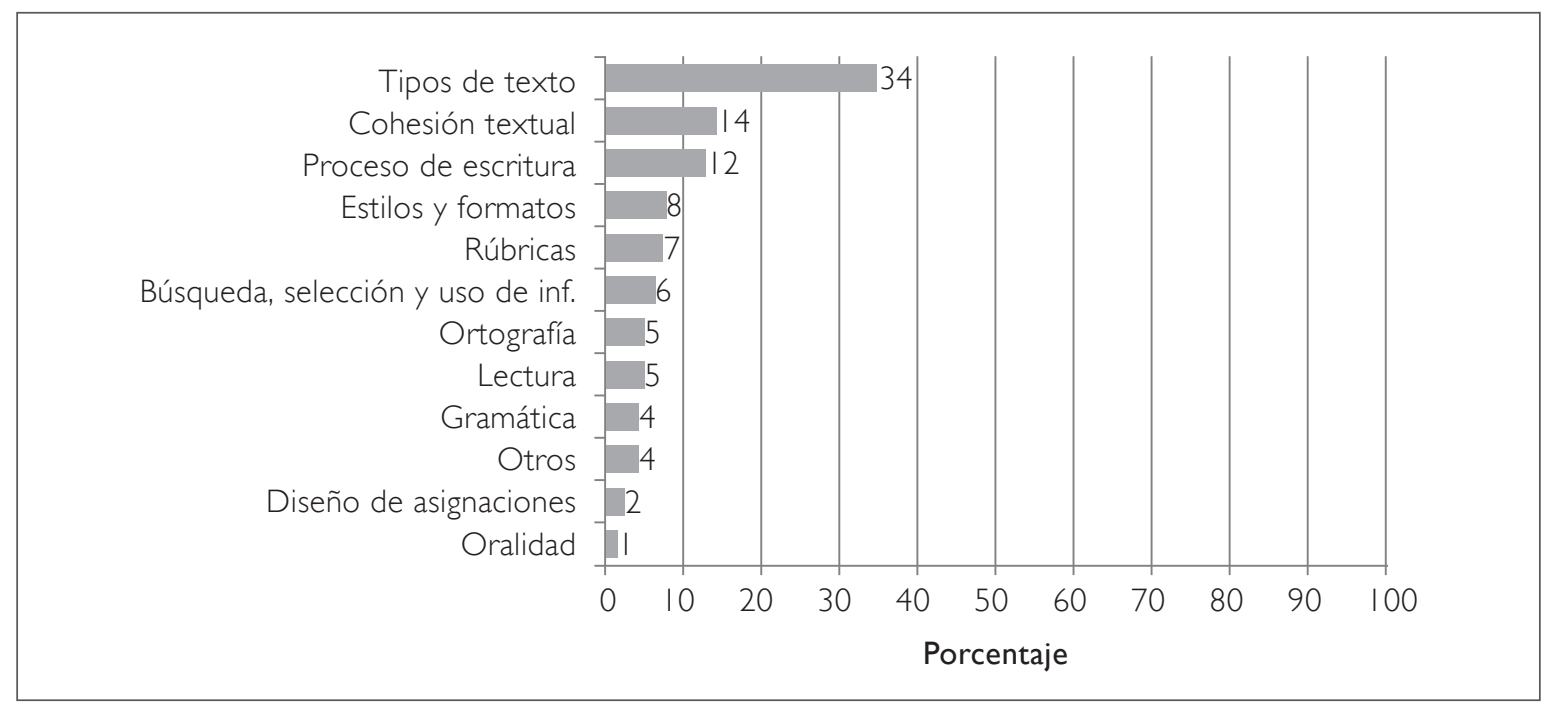

Gráfico 2. Contenidos de los recursos. Fuente propia.

El 34\% de los recursos analizados se orientan a la caracterización de tipologías textuales, entre ellos los más comunes son el ensayo académico y la reseña. A pesar de las diferentes denominaciones 
de la categoría (géneros, tipos, modelos), las páginas presentan clasificaciones muy similares en sus recursos. Los contenidos también comparten rasgos básicos. Por ejemplo, en "reseña", los recursos coinciden en indicar que se presenta una síntesis de una obra o producto cultural, a la vez que hace una valoración crítica. Los distintos recursos sobre reseña recomiendan una contextualización sobre el autor y producción de la obra, así como relaciones de intertextualidad para poder hacer un comentario crítico sobre su contenido o forma.

En esta categoría llama la atención, como lo señala Narváez²', que la mayoría de recursos sobre tipologías textuales promueven prácticas de escritura estandarizadas y homogéneas, pues las instrucciones para escribir los textos y los ejemplos que se presentan en los recursos parecen mostrar que hay una sola forma de escribir en la universidad. Se desconoce, implícitamente, la naturaleza de la escritura académica, que como plantea Carlino ${ }^{22}$, involucra una gran diversidad de prácticas relacionadas con los modos discursivos de las disciplinas. Pocos recursos atienden a formas de escribir específicas en las distintas áreas del conocimiento. Una de las excepciones es un libro de acceso gratuito publicado por el PRODEAC de Argentina que caracteriza, por ejemplo, el análisis de caso en educación o la evaluación de producto en ingeniería. Otros recursos que suelen mostrar diferencias en los modos de escribir en las disciplinas son los relacionados con los informes, en los que se diferencia entre informe financiero, informe administrativo, informe de laboratorio, etc.

Además de los tipos de texto, los recursos analizados se centran en aspectos como la cohesión textual (14\%) y el proceso de escritura ( 12\%). En cohesión textual, los temas más comunes son el párrafo, los conectores, los mecanismos léxicos y gramaticales para mantener los referentes y la puntuación. Por su parte, los recursos relacionados con el proceso de escritura enfatizan en que los autores planeen sus textos, construyan varios borradores, revisen y ajusten sus escritos antes de entregarlos al lector. La mayoría de recursos sobre el proceso de escritura insisten en que el proceso no es lineal, sino recurrente, y establecen diferencias entre revisar y corregir un texto. La revisión se relaciona con aspectos más amplios como el cumplimiento del propósito o el ajuste a la audiencia, mientras la corrección se relaciona con la edición del texto.

Varios centros y programas ofrecen recursos que sintetizan algunas normas y estilos de citación (8\%), orientaciones sobre cómo buscar y seleccionar fuentes confiables de información (6\%), y manuales ortográficos y gramaticales muy completos. Esta información suele ajustarse a audiencias no especializadas y facilita a los usuarios la labor de producción de textos ${ }^{23}$.

Los temas menos abordados en los recursos son la oralidad académica (1\%) y el diseño de asignaciones de lectura y escritura (2\%). Este último es un tema de vital importancia, debido a que

2I Narváez, Elizabeth. Iniciativas de enseñanza de la escritura en países latinoamericanos: Tendencias emergentes de las páginas web de centros de escritura y programas de escritura, 2013. Memorias del I Congreso Latinoamericano de Centros de Escritura. Recuperado de: https://sites.google.com/site/congresocentrosdeescritura/file-cabinet

22 Carlino, Paula. "Alfabetización académica diez años después". Revista Mexicana de Investigación Educativa, vol. I8, n 57 (20 I3), págs. 355-381.

23 Cassany, Daniel. En línea: Leer y escribir en la red. España: Anagrama, 2013. 
las transformaciones en los modos de orientar la lectura y la escritura por parte de los profesores de las disciplinas pueden tener una gran incidencia en las prácticas de los estudiantes universitarios ${ }^{24}$ y, como se evidencia, es tema muy poco abordado en los recursos.

\section{Formatos de información digital}

Esta categoría hace referencia a las modalidades de representación de la información que predominan en los recursos.

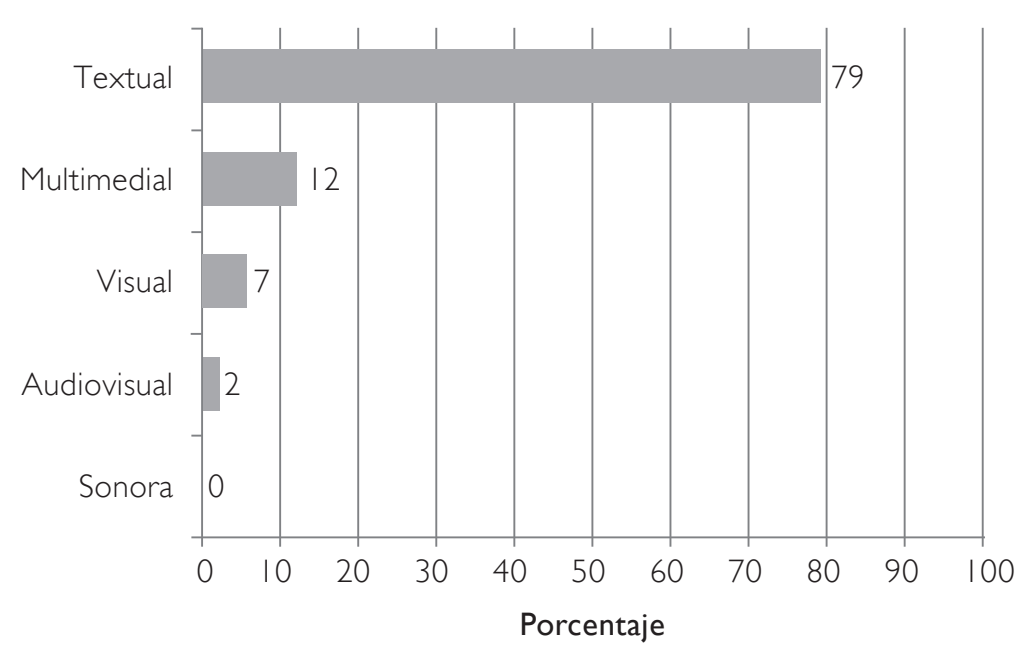

Gráfico 3: formatos de información digital. Fuente propia.

La modalidad de información digital más común es el texto (79\%). La mayoría de estos textos se presentan en formato PDF (63\%) y en Word (27\%) y pueden ser descargados. Aunque estos recursos presentan información predominantemente textual, varios de ellos incluyen algún elemento gráfico de apoyo (imagen, tabla) o rasgos paratextuales (subtítulos, subrayado, negrita) que ayudan a destacar ideas. La extensión de los recursos textuales es variada, el promedio es de cinco páginas, pero el rango es muy amplio: entre I y 58 páginas. Los textos más extensos son aquellos relacionados con estilos y formatos de citación, como normas APA y MLA.

24 Kuriloff, Peshe. "The Writing Consultant: Collaboration and Team Teaching". In McLeod, Susan \& Soven, Margot (eds.). Writing Across the Curriculum: A Guide to Developing Programs. California, USA: WAC Clearinghouse Landmark Publications in Writing Studies, Sage Publications. 
El formato multimedial ( $12 \%$ ) se caracteriza por permitir una mayor interacción del usuario con el recurso. Los más sencillos implican que el usuario elija las rutas de navegación y se encuentre con modalidades textuales, visuales y audiovisuales, mientras que otros tienen unas complejas plataformas en las que el usuario, además de visualizar los contenidos, puede realizar ejercicios y recibir realimentaciones automáticas $^{25}$. En esta categoría se destacan los recursos propuestos por el CREA del Tecnológico de Monterrey y el Centro de Escritura de la UPAEP de México.

La modalidad visual, como componente privilegiado en la representación de información, aparece muy poco, casi siempre aparece como auxiliar de otros formatos. En esta modalidad se destacan las infografías propuestas por el Centro de Escritura Javeriano de la Pontificia Universidad Javeriana Cali.

Apenas se identificó un $2 \%$ de recursos en modalidad audiovisual, con algunos videos alojados en YouTube. En la muestra no se encontraron recursos en modalidad predominantemente sonora, posiblemente porque las otras modalidades son más pertinentes para trabajar en temas de escritura académica.

Respecto a los formatos de información digital, McKinney ${ }^{26}$ resalta la importancia de producir recursos sobre escritura en distintas modalidades, ya que como plantea el Committee for Effective Practices in Online Writing Instruction ${ }^{27}$ esto permite no solo una mejor adaptación de los centros a las demandas de la sociedad del conocimiento, sino que también se promueven prácticas de inclusión educativa, en las que se facilita la información en canales que pueden llegar a cualquier población (con y sin discapacidad) y atienden a las posibles preferencias de los usuarios. Es importante considerar los cambios históricos y entender las nuevas literacidades como oportunidades para los centros y programas en sus diferentes servicios, tanto en los recursos o materiales autónomos que ofrecen en la web, como en las interacciones en las que median los tutores (tutorías presenciales y virtuales) ${ }^{28}$.

En una línea similar, Cacheiro ${ }^{29}$ propone aprovechar las características de las pantallas para variar los formatos de presentación de la información y para favorecer que los usuarios se involucren e interactúen mucho más con los contenidos. Muchos recursos están alojados en páginas web, pero son documentos descargables en formato PDF o Word y no se aprovechan las características de hipertextualidad y multimodalidad. Las pantallas permiten que algunos ejercicios puedan ser interactivos y proporcionen una respuesta básica, lo que conduce a una flexibilización de los recursos y una personalización de los contenidos adaptados a las características de los usuarios.

25 Ministerio de Educación Nacional de Colombia. Recursos educativos digitales abiertos. Bogotá: Colección Sistema Nacional de Innovación Educativa con uso de TIC, 2012.

26 McKinney, Jackie. "New Media Matters: Tutoring in the Late Age of Print" (p. 344-36I). In Murphy, Christina \& Sherwwood, Steve. The St. Martin's sourcebook for writing tutors. Fourth edition. Boston: Bedford/St. Martin's Press, 201 I .

27 Committee for Effective Practices in Online Writing Instruction. 20 I3. Consultado I 5 de octubre de 20 I5. Recuperado de: http:// www.ncte.org/cccc/committees/owi

28 López-Gil, Karen. "Tutorías virtuales: retos y oportunidades en los centros de escritura de América Latina". En Molina, Violeta. Panorama de los centros y programas de escritura en Latinoamérica. Cali, Colombia: Sello Editorial Javeriano, 20I5.

29 Cacheiro, María. "Recursos educativos TIC de información, colaboración y aprendizaje". Píxel-Bit, Revista de Medios y Educación, n³9, Julio (201 I), págs. $69-81$. 


\section{Grado de interactividad de los recursos}

Esta categoría se refiere al grado en que el usuario puede influir en el comportamiento del recurso.

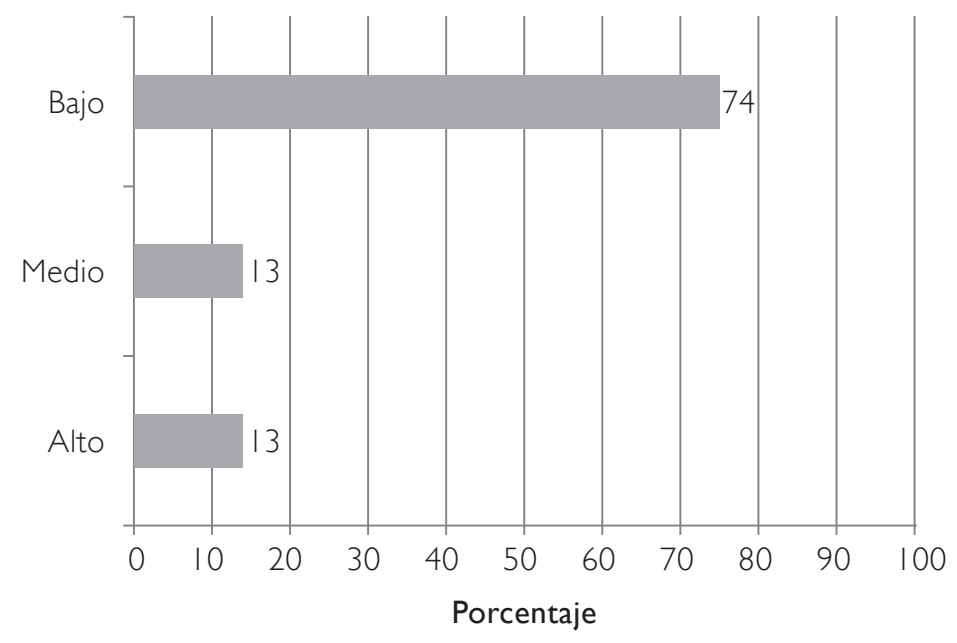

Gráfico 4. Grado de interactividad de los recursos. Fuente propia.

El 74\% de los recursos analizados tiene un nivel de interacción bajo. Esto se evidencia en que la mayoría de estos recursos solo requieren que el usuario navegue en la página y descargue un documento en modalidad textual. Los recursos con un nivel de interacción media ofrecen al usuario más posibilidades de tomar decisiones en las rutas de navegación; en estos se destacan los recursos en formato hipertextual (página web).

Los recursos con un grado de interactividad alta permiten, además de navegar, responder ante demandas específicas y recibir una realimentación. Se destacan en esta última modalidad algunos recursos del blog de redacción de la Pontificia Universidad Católica del Perú. Este blog tiene ejercicios interactivos con respuestas cerradas y también permite que el usuario valore la calidad y pertinencia de los materiales publicados a través de comentarios y calificación de cada recurso. Los administradores de la página suelen dar respuesta a los comentarios de los usuarios en corto tiempo.

Al retomar la clasificación de los recursos educativos planteada por Barbera y Rochera ${ }^{30}$, se puede identificar que la mayoría de los materiales compartidos por los centros y programas de escritura de Latinoamérica son reproductivos/informativos, esto indica que el usuario puede acceder a una

30 Barbera, Elena y Rochera, María José. "Los entornos virtuales de aprendizaje basados en el diseño de materiales autosuficientes y el aprendizaje autodirigido" (págs. 173- 193). En Coll, César y Monereo, Carles (Eds.) Psicología de la educación virtual. Madrid: Ediciones Morata, 2008. 
información o contenido estructurado a través del recurso, pero tiene poca interacción y realimentación cuando lo usa.

\section{Público al que se dirigen}

Esta categoría se refiere a la audiencia a la que van dirigidos los recursos. Esta audiencia puede ser explícita en el recurso o en la página (ejemplo: sección de recursos para estudiantes) o puede inferirse a partir de los contenidos y de su forma de organización.

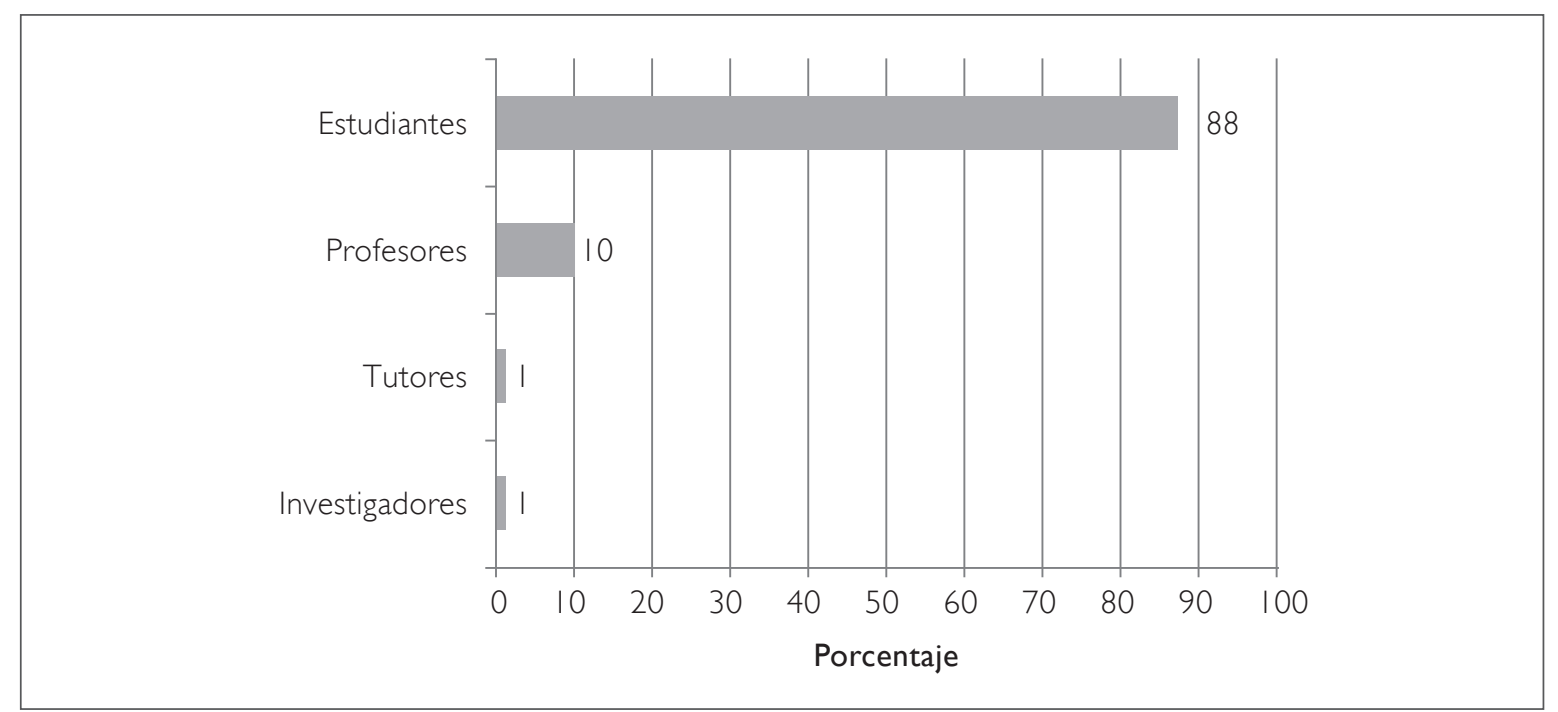

Gráfico 5: público al que se dirigen los recursos. Fuente propia.

El gráfico 5 evidencia que la mayoría de recursos (88\%) se dirige a estudiantes, aunque por las características de sus contenidos pueden ser utilizados por cualquier usuario que esté en rol de escritor.

Entre los recursos dirigidos específicamente a profesores ( $10 \%$ ) se destacan una multimedia educativa del Centro de Escritura Javeriano sobre cómo orientar la lectura y la escritura en asignaturas disciplinares, las matrices de evaluación de textos planteadas por el Centro de Español de la Universidad de los Andes y los recursos de apoyo a profesores del Centro de Redacción Multidisciplinario de la Universidad Interamericana de Puerto Rico.

Muy pocos recursos se dirigen a tutores (1\%). El Centro de Redacción Multidisciplinario ofrece recursos dirigidos especialmente a esta audiencia, en los que llaman la atención un manual en inglés y otro en español sobre las funciones de los tutores y sobre cómo orientar el proceso de escritura. La mayoría de los centros de escritura cuentan con tutores y estos tutores requieren de procesos 
de formación continua y aprendizaje autogestionado, por lo que crear recursos específicos para esta población puede ser otra oportunidad importante para los centros y programas de Latinoamérica.

Finalmente, se encuentran pocos recursos dirigidos a investigadores ( $1 \%)$. Estos recursos abordan temas como la presentación de proyectos de investigación o la escritura de artículos científicos.

\section{Licencias y permisos de uso de los recursos}

Esta categoría hace referencia a las condiciones de acceso y permisos de distribución, uso y adaptación de los recursos otorgados por el autor.

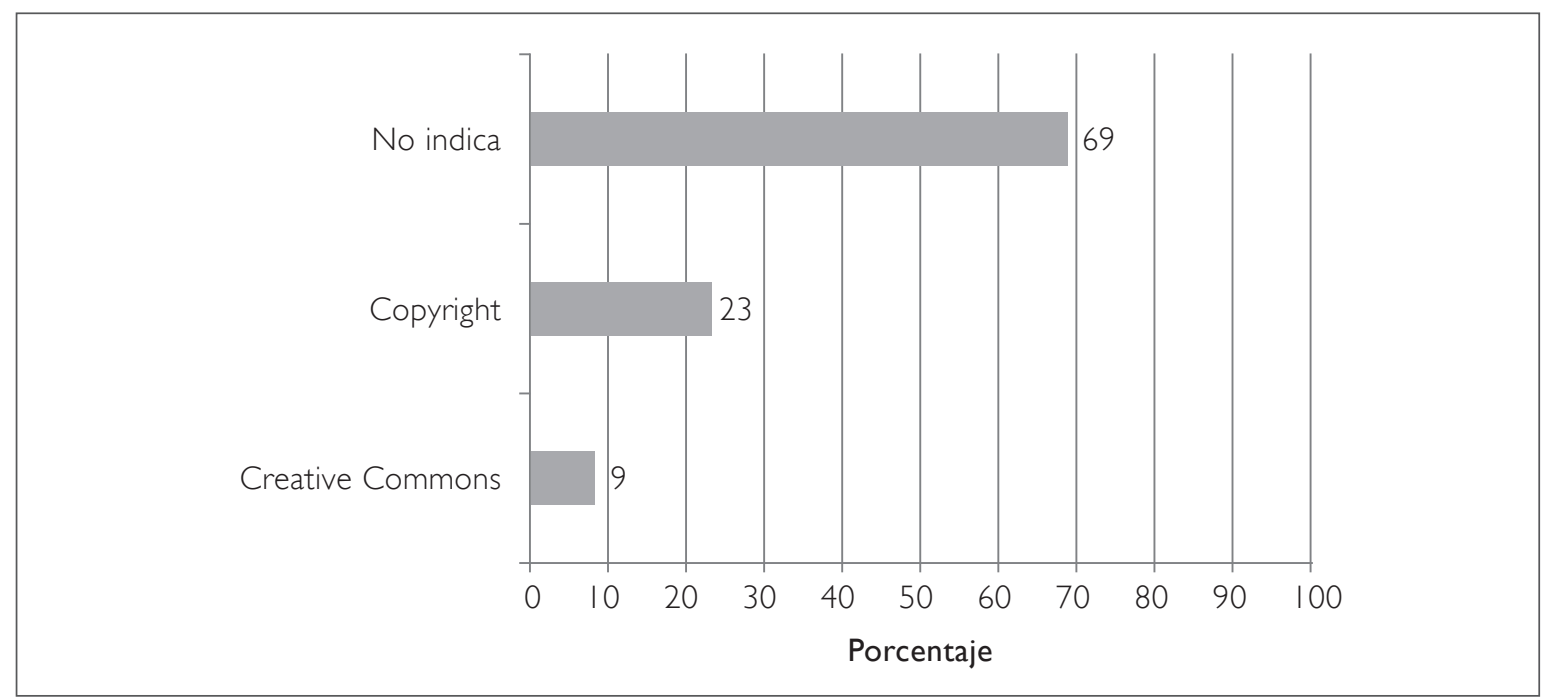

Gráfico 6: licencias y permisos de uso. Fuente propia.

Como se evidencia en el gráfico 6, el 69\% de los recursos analizados no tienen explícitas sus condiciones de acceso y permisos de uso. $23 \%$ de los recursos tienen Copyright, lo que limita sus condiciones de reproducción y uso. Apenas un $9 \%$ de los recursos tiene licencia Creative Commons. De esta manera, se identifica que la mayoría de los recursos compartidos por los centros y programas de escritura de Latinoamérica no pueden ser modificados ni reutilizados por otros.

Los recursos identificados tampoco hacen parte de repositorios, ni cuentan con metadatos (palabras clave, nivel educativo, fecha de creación, modalidad de representación de la información, nivel de interactividad, etc.) que permitan un acceso más rápido y ágil por parte de los usuarios.

Estos resultados pueden convertirse en una oportunidad para el trabajo en red, ya que la creación de recursos educativos digitales con licencias libres y registrados en repositorios puede potenciar 
la oferta formativa digital en escritura académica. Si bien algunos recursos se apoyan en teorías y enfoques distintos, la mayoría comparte rasgos básicos. Por ejemplo, la mayoría de recursos analizados promueve la concepción de la escritura como proceso, la identificación de situación retórica como un aspecto clave de la escritura, la identificación de los géneros académicos en las disciplinas, el conocimiento de normas básicas de ortografía y gramática, etc.

\section{Referencias bibliográficas identificadas en los recursos}

Esta categoría se refiere a la identificación de relaciones de intertextualidad y fundamentación de los recursos a través de mecanismos de citación y uso de referencias bibliográficas.

Se identificó que 47\% de los recursos no tenían referencias bibliográficas. En el 53\% restante, se identifican como más frecuentes aquellas referencias que corresponden a manuales de escritura o redacción (ejemplos: Saber Escribir del Instituto Cervantes, la Cocina de la escritura y Describir el escribir de Daniel Cassany), libros de ortografía y gramática de la Real Academia Española y algunas traducciones y adaptaciones de recursos de centros de escritura de Estados Unidos como Purdue, North Caroline, MTI o Harvard.

Las referencias que suelen usarse como sustento en estos recursos apoyan la representación de la escritura como un conjunto de prácticas homogéneas y transferibles a distintos contextos. Por otro lado, la ausencia de referencias en los recursos cuestiona el ejemplo que los centros y programas ofrecemos a los usuarios, pues la escritura académica se caracteriza, entre muchos otros aspectos, por el establecimiento de relaciones de intertextualidad y por el uso riguroso de estilos y formatos de citación.

\section{Enlaces a otros centros y programas de escritura}

De las 17 páginas revisadas, 5 tienen enlaces que remiten a sus usuarios a los recursos disponibles en otros centros y programas de escritura. Sin embargo, la mayoría de páginas (12) incluyen enlaces de interés a portales relacionados con la escritura en general, como la página de la Real Academia Española, el Centro Virtual Cervantes y la Fundación para el Español Urgente- Fundéu.

\section{Conclusiones}

Se encontró que la mitad de los centros y programas que cuentan con páginas web comparten recursos elaborados por ellos mismos. Entre las características más importantes de estos recursos están que la mayoría son expositivo-instructivos, es decir que buscan proporcionar información sobre cómo realizar un procedimiento, por ejemplo, cómo organizar las referencias bibliográficas. El tema más recurrente en los recursos analizados es el de tipologías textuales, entre estas las más frecuentes son el ensayo y la reseña académica. El formato que predomina es el textual y se abordan poco otras modalidades de 
representación de la información. El grado de interactividad de los recursos es bajo, pues están diseñados para que el usuario los consulte o navegue, pero no para que te interactúe con los contenidos. Los estudiantes son el público privilegiado. La mayoría de recursos no indican explícitamente sus condiciones de uso o están protegidos con derechos de autor. Cerca de la mitad de los recursos no incluyen referencias bibliográficas de apoyo.

A partir de estos resultados, se puede concluir que los recursos educativos sobre escritura compartidos por los centros y programas están promoviendo, implícitamente, la visión de la escritura como una práctica homogénea y transferible a cualquier contexto, concepción que no es coherente con las perspectivas que sustentan a la mayoría de los centros y programas de Latinoamérica ${ }^{31}$ (Alfabetización Académica, Escritura a través del Currículo, Escritura en las Disciplinas, etc.). En estas perspectivas se plantea que la escritura en la universidad involucra una gran diversidad de prácticas que se relacionan estrechamente con los modos de construir, transformar y comunicar los conocimientos en las distintas áreas. En consecuencia, se esperaría que los recursos elaborados por los centros y programas ayuden a los usuarios a identificar y a practicar los rasgos propios de la escritura en las disciplinas, en los distintos momentos del proceso formativo.

Por otra parte, cabe resaltar que en la mayoría de los recursos se promueve la concepción de la escritura como un proceso y no solo como un producto y se ofrecen distintas estrategias para que los usuarios lleven a cabo las distintas fases de la composición, sin que esto implique un desarrollo estrictamente lineal. De igual forma, en los recursos se hace énfasis en la estructura básica de los textos y en mecanismos de cohesión y coherencia. Esta información constituye un valioso aporte para los usuarios que se están acercando a la producción de textos en la universidad.

Los centros y programas podrían concentrarse en la elaboración de recursos que respondan de forma más directa a las nociones de escritura que quieran compartir, en la medida en que puedan apoyarse en el trabajo que otros han desarrollado; por ejemplo, hay varios recursos sobre formatos y estilos de citación, generalidades sobre la escritura de textos argumentativos, ortografía, cohesión textual, etc., pero hay pocos que se centren en los distintos modos de escribir en las disciplinas y en las transformaciones que van teniendo los estudiantes cuando se introducen, practican y se consolidan como miembros de una comunidad discursiva.

Ayudaría mostrar a los usuarios de cada página de centro o programa la existencia de otros recursos de escritura en los que pueden apoyarse. Además de compartir información, la posibilidad de convertir los recursos actuales en recursos con licencias abiertas favorecería la reutilización de los materiales y su inclusión en un repositorio global que facilite su consulta. De esta manera, la elaboración de recursos se puede concentrar en la difusión de prácticas más heterogéneas de escritura.

3I Narváez, Elizabeth. Iniciativas de enseñanza de la escritura en países latinoamericanos: Tendencias emergentes de las páginas web de centros de escritura y programas de escritura, 20 I3. Memorias del I Congreso Latinoamericano de Centros de Escritura. Recuperado de: https://sites.google.com/site/congresocentrosdeescritura/file-cabinet 
Esta es una oportunidad importante, debido a que en la actualidad hay muchos recursos disponibles en la red - si se introduce en el buscador de Google "cómo escribir" resultan cercan de 39.000.000 de entradas-, por lo que un repositorio, o al menos un conjunto de recursos conectados entre sí, podría ayudar a los usuarios de las universidades a tener mayor certeza de la confiabilidad de las fuentes que están usando y que los orientan en la producción de sus textos académicos. Además, como plantea la UNESCO ${ }^{32}$, la creación de recursos educativos abiertos es uno de los principales lineamientos para la consolidación de la educación superior, pues estos materiales aportan a la democratización del conocimiento y al derecho a acceder y a usar información de calidad. Los centros y programas debemos reconocer que una vez los recursos están en la web son públicos y pueden ser consultados por cualquier persona, lo que implica una importante responsabilidad en el propósito formativo que nos planteamos y en los diseños que empleamos.

Finalmente, valdría la pena explorar las percepciones que tienen los usuarios sobre los recursos digitales ${ }^{33}$, en este caso compartidos por los centros y programas y, a partir de los usos reales, contar con elementos que permitan fortalecer la oferta formativa en escritura académica.

\section{Bibliografia}

Álvarez, Teodoro y Ramírez, Roberto. "El texto expositivo y su escritura". Revista Folios, julio-diciembre (20 I0), págs. 73-88.

Barbera, Elena y Rochera, María José. "Los entornos virtuales de aprendizaje basados en el diseño de materiales autosuficientes y el aprendizaje autodirigido" (págs. 173-193). En Coll, César y Monereo, Carles (Eds.) Psicología de la educación virtual. Madrid: Ediciones Morata, 2008.

Blair, Kristine. "Teaching Multimodal Assignments in OWI Contexts" (págs. 47I-49I). In Hewett, Beth and DePew, Kevin (Eds.) Foundational Practices of Online Writing Instruction. Perspectives on Writing. Fort Collins, Colorado: The WAC Clearinghouse and Parlor Press, 2015.

Cacheiro, María. "Recursos educativos TIC de información, colaboración y aprendizaje". Píxel-Bit, Revista de Medios y Educación, n³9, Julio (2011), págs. 69 - 81 .

Carlino, Paula. "Alfabetización académica diez años después". Revista Mexicana de Investigación Educativa, vol. I8, n 57 (20 I3), págs. 355-381.

Cassany, Daniel. En línea: Leer y escribir en la red. España: Anagrama, 2013.

Colectivo Educación Infantil y TIC. Recursos educativos digitales para la educación infantil (REDEI). Revista Zona Próxima, $n^{\circ} 20$, enero-junio (2014), págs. I-2I.

32 UNESCO. A Basic Guide to Open Educational Resources: Frequently asked questions. 20 I I. Recuperado de: http://unesdoc.unesco. org/images/0023/002328/232855s.pdf

33 Rodríguez, Ninel; Telles, Ana; Vértiz, María y Sánchez, Abigaíl. "Estudio de casos: REA (recursos educativos abiertos) en clases de Historia de México". Magis, Revista Internacional de Investigación en Educación, vol. 3, n 5 (20 I0) págs, I47-I66. 
Committee for Effective Practices in Online Writing Instruction. 2013. Consultado I5 de octubre de 2015. Recuperado de: http://www.ncte.org/cccc/committees/owi

Contreras-Espinosa, Ruth. "Recursos educativos abiertos: Una iniciativa con barreras aún por superar". Revista Apertura, vol. 2, nº 2 (20।0).

Kuriloff, Peshe. "The Writing Consultant: Collaboration and Team Teaching". In McLeod, Susan \& Soven, Margot (eds.). Writing Across the Curriculum: A Guide to Developing Programs. California, USA: WAC Clearinghouse Landmark Publications in Writing Studies, Sage Publications.

Lamorux, Tomas. Recursos audiovisuales en Internet diseñados para el aprendizaje del francés como lengua extranjera (FLE) dirigido a un público adulto. Tesis doctoral. España: Universidad Nacional de Educación a Distancia, 20 I4.

López-Gil, Karen. "Tutorías virtuales: retos y oportunidades en los centros de escritura de América Latina". En Molina, Violeta. Panorama de los centros y programas de escritura en Latinoamérica. Cali, Colombia: Sello Editorial Javeriano, 2015.

Martínez, Diane \& Olsen, Leslie. "Online Writing Labs" (págs. I83-210). In Hewett, Beth and DePew, Kevin (Eds.) Foundational Practices of Online Writing Instruction. Perspectives on Writing. Fort Collins, Colorado: The WAC Clearinghouse and Parlor Press, 2015.

Ministerio de Educación Nacional de Colombia. Recursos educativos digitales abiertos. Bogotá: Colección Sistema Nacional de Innovación Educativa con uso de TIC, 2012.

Molina, Violeta. Panorama de los centros y programas de escritura en Latinoamérica. Cali, Colombia: Sello Editorial Javeriano, 2015.

Narváez, Elizabeth. Iniciativas de enseñanza de la escritura en países latinoamericanos: Tendencias emergentes de las páginas web de centros de escritura y programas de escritura, 20 I3. Memorias del I Congreso Latinoamericano de Centros de Escritura. Recuperado de: https://sites.google.com/site/congresocentrosdeescritura/file-cabinet

Núñez, Juan. "Una aproximación a los centros de escritura en Iberoamérica”. Revista Legenda, vol. 17, n 7 (20।3), págs. 62-102.

McKinney, Jackie. "New Media Matters: Tutoring in the Late Age of Print" (págs. 344-36I). In Murphy, Christina \& Sherwwood, Steve. The St. Martin's sourcebook for writing tutors. Fourth edition. Boston: Bedford/St. Martin's Press, 2011.

Pinto, María. "Evaluación y mejora de la calidad de los recursos educativos electrónicos en el ámbito universitario español desde un enfoque documental". Ibersid, vol. 4 (20 I0), págs. I05-I I 6.

Pinto, María y Gómez-Camarero, Carmen. "Propuesta de criterios e indicadores internacionales para la evaluación de los recursos educativos electrónicos”. Ibersid, vol. 5 (201 I), págs. 81-87.

Red Latinoamericana de Centros y Programas de Escritura. Integrantes. 20I5. Consultado 25 de mayo de 2015 Recuperado de https://sites.google.com/site/redlacpe/integrantes

Santos, Gema; Ferrán, Nuria y Abadán, Ernest. "Recursos educativos abiertos: repositorios y usos". El profesional de la información, vol. 21, nº 2 (2012), págs. 136-145.

UNESCO. A Basic Guide to Open Educational Resources: Frequently asked questions. 201 I. Recuperado de: http:// unesdoc.unesco.org/images/0023/002328/232855s.pdf 
UNESCO. Declaración de París de 2012 sobre los REA. 20 I2. Recuperado de: http://www.unesco.org/new/fileadmin/ MULTIMEDIA/HQ/Cl/Cl/pdf/Events/Spanish_Paris OER Declaration.pdf

Rodríguez, Ninel; Telles, Ana; Vértiz, María y Sánchez, Abigaíl. "Estudio de casos: REA (recursos educativos abiertos) en clases de Historia de México". Magis, Revista Internacional de Investigación en Educación, vol. 3, n 5 (2010) págs. 147- 66.

\section{Páginas web de los centros y programas de escritura}

Blog de redacción del Programa de Comunicación Académica de la Pontificia Universidad Católica del Perú. http:// blog.pucp.edu.pe/blog/blogderedaccion/

Centro de Escritura Javeriano de la Pontificia Universidad Javeriana Cali. http://centrodeescritura.javerianacali.edu.co/ Centro de Lectura y Redacción de la Universidad del Turabo. http://centrodelecturayredaccion.blogspot.com.co/

Centro de Escritura Académica y Pensamiento Crítico de la Universidad de las Américas de Puebla. http://www. udlap.mx/centrodeescritural

Centro de Redacción Multidisciplinario de la Universidad Interamericana de Puerto Rico. http://www.metro.inter. edu/facultad/esthumanisticos/crem intro.asp

Centro de Escritura Universitaria de la Universidad de San Andrés. http://live.vl .udesa.edu.ar/Unidades-Academicas/ departamentos-y-escuelas/Humanidades/Centro-de-Escritura-Universitaria

Centro de Recursos para la Escritura Académica (CREA) del Tecnológico de Monterrey. http://sitios.ruv.itesm. mx/portales/creal

Programa de Escritura Centro de Español de la Universidad de los Andes. http://programadeescritura.uniandes.edu.co/

Centro de Aprendizaje, Redacción y Lenguas del Instituto Tecnológico Autónomo de México. http://carle.itam.mx/

Centro de Escritura de la Universidad del Cauca. http://www.unicauca.edu.co/centroescritura/

Centro Virtual de Escritura de la Universidad de Buenos Aires. https://centrodeescrituravirtual.wordpress.com/

DIGA-Centro de apoyo para la lectura, la oralidad y la escritura del Centro de Estudios Superiores de Administración. http://www.cesa.edu.co/El-Cesa/DIGA.aspx

Centro de Recursos para el Éxito Estudiantil - CREE y programa Eficacia Comunicativa de la Universidad del Norte. http://www.uninorte.edu.co/web/centro-de-recursos-para-el-exito-estudiantil-cree/centro-de-recursos-parael-exito-estudiantil

Centro de Escritura de la Universidad Popular Autónoma del Estado de Puebla. www.upaep.mx/centrodeescritura/ Programa de Lectura y Escritura Académicas de la Pontificia Universidad Católica de Chile. http://www.pleauc.cl/ Centro de Escritura CEDES de la Universidad Católica del Uruguay. http://www.ucu.edu.uy/es/cedes\#.VuC2VPKrKM8 Programa de desarrollo de habilidades de lectura y escritura académica a lo largo de la carrera (PRODEAC) de la Universidad Nacional de General Sarmiento. http://www.ungs.edu.ar/prodeacl 


\section{Anexo I: rejilla de análisis de recursos educativos digitales compartidos por centros y programas de escritura de América Latina}

\begin{tabular}{|l|l|}
\hline & I. Identificación del recurso \\
\hline 1.I Nombre del recurso: & \\
\hline 1.2 Centro o programa: & \\
\hline 1.3 Propósito del centro o programa: & \\
\hline 1.4 País: & \\
\hline 1.5 Idioma del recurso: & \\
\hline 1.6 Público al que se dirige: & \\
\hline 1.7 Enlace: & \\
\hline
\end{tabular}

\begin{tabular}{|c|c|}
\hline \multicolumn{2}{|r|}{ 2. Tipo de recurso } \\
\hline 2.I Gestión del aprendizaje & Autogestionado ( ) Mediado por tutor ( ) \\
\hline & $\begin{array}{l}\text { Explicativo/instructivo () Práctico/ejercicio () } \\
\text { Argumentativo () Rejilla de evaluación( ) }\end{array}$ \\
\hline 2.2 Propósito & $\begin{array}{l}\text { Estrategias discursivas en recursos explicativos: } \\
\text { Definición/descripción ( ) } \\
\text { Definición/descripción más ejemplo ( ) } \\
\text { Ejemplo y ejercicio ( ) } \\
\text { Clasificación ( ) } \\
\text { Fases/procesos ( ) } \\
\text { llustración ( ) } \\
\text { Comparación/contraste () }\end{array}$ \\
\hline 2.3 Tema del recurso & R \\
\hline 2.4 Formato de información digital & $\begin{array}{l}\text { Textual () Visual () Sonoro ( ) } \\
\text { Audiovisual ( ) Multimedial () }\end{array}$ \\
\hline 2.5 Formato de archivo & $\begin{array}{l}\text { Documento PDF ( ) Documento Word ( ) } \\
\text { Diapositivas PDF ( ) Diapositivas PPT ( ) } \\
\text { Página web ( ) Video ( ) Imagen ( ) } \\
\text { Zip/Rar ( ) Otro, cuál }\end{array}$ \\
\hline 2.6 Modalidad de consulta & En línea () Descargable () Aplicación móvil () Nube () \\
\hline 2.7 Nivel de interactividad & Bajo () Medio () Alto () \\
\hline
\end{tabular}




\begin{tabular}{|l|l|}
\hline \multicolumn{2}{|l|}{ 3. Contenidos del recurso } \\
\hline 3.1 Propósito & R/ \\
\hline 3.2 Explicita su enfoque & No ( ) Sí ( ), cuál \\
\hline 3.3 Estructura del recurso & R \\
\hline & No ( ) Al menos una ( ) Entre dos y cinco ( ) \\
3.4 Referencias bibliográficas & Entre cinco y diez ( ) Más de diez ( ) \\
& Cuáles__ \\
\hline
\end{tabular}

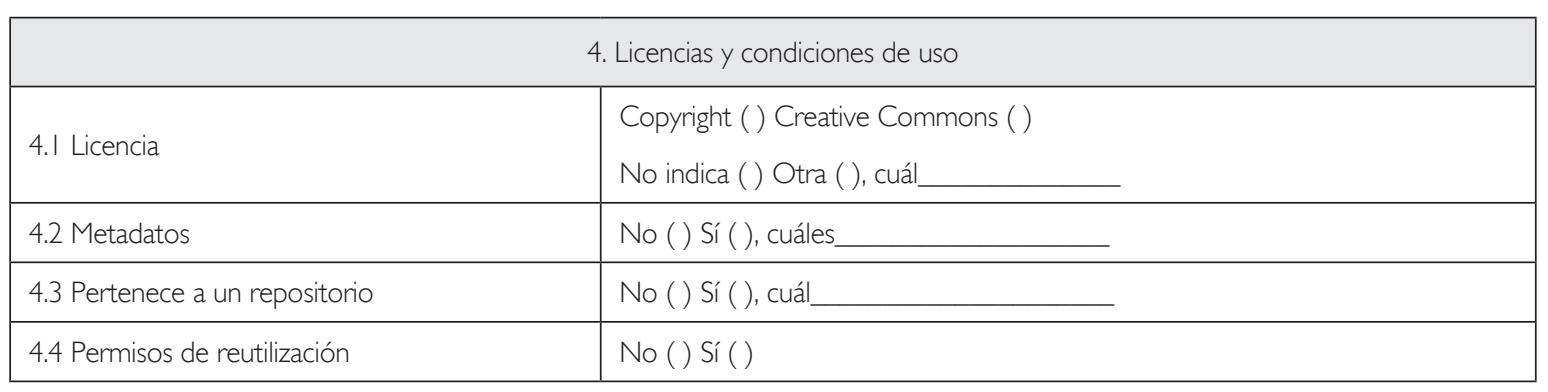

\begin{tabular}{|l|l|}
\hline \multicolumn{2}{|c|}{ 5. Aspectos técnicos } \\
\hline 5.I Plugins adicionales (lava, Flash player) & No ( ) Sí ( ), cuáles \\
\hline 5.2 Navegabilidad & Sencilla ( ) Dificultad media ( ) Compleja ( ) \\
\hline 5.3 Requiere manual de instrucciones & No ( ) Sí ( ) \\
\hline $\begin{array}{l}\text { 5.5 Accesibilidad, pertinencia de las fuentes } \\
\text { (tamaño, letra, contraste) }\end{array}$ & No ( ) Sí ( ) \\
\hline 5.5 Diseño estético y llamativo & No ( ) Sí ( ) \\
\hline
\end{tabular}

\begin{tabular}{|l|l|}
\hline \multicolumn{2}{|l|}{ 6. Relación con otros recursos } \\
\hline 6.I Enlaces a otros centros y programas & No ( ) Sí ( ) \\
\hline 6.2 Enlaces a otras páginas & Cuáles_ \\
\hline & No ( ) Sí ( ) \\
\hline
\end{tabular}

